\title{
Image De-Noising and Compression Using Statistical based Thresholding in 2-D Discrete Wavelet Transform
}

\author{
Qazi Mazhar \\ Military College of Signals, \\ National University of Sciences and Technology \\ Rawalpindi, Pakistan \\ Adil Masood Siddique \\ Military College of Signals, \\ National University of Sciences and Technology \\ Rawalpindi, Pakistan
}

\author{
Imran Touqir \\ Military College of Signals, \\ National University of Sciences and Technology \\ Rawalpindi, Pakistan \\ Adnan Ahmad Khan \\ Military College of Signals, \\ National University of Sciences and Technology \\ Islamabad, Pakistan
}

\begin{abstract}
Images are very good information carriers but they depart from their original condition during transmission and are corrupted by different kind of noise. The purpose is to remove the noisy coefficients such that minimum amount of information is lost and maximum amount of noise is suppressed or reduced. We considered Generalized Gaussian distribution for modeling of noise. In the proposed technique, statistical thresholding methods are used for the estimation of threshold value while Biorthogonal wavelet has been envisaged for image decomposition and reconstruction. A qualitative and quantitative analysis of thresholding methods on different images shows significant results for statistical thresholding methods based on objective and subjective quality as compared to other de-noising methods.
\end{abstract}

Keywords-Wavelet Thresholding; statistical Thresholding Image De-noising; Image Compression; Wavelet Sub-band Thresholding

\section{INTRODUCTION}

In the era of this digital world the use of digital images is greatly increased. Digital images are used in satellite, medical, radar, computer vision and pattern recognition. All these images are in digital form and noise is introduced in it during transmission, acquisition and processing. These images need to be de-noised before it is used in some kind of application. The goal of digital image de-noising is to restore the original image from a noise contaminated image and to preserve the important features of the image during the dropping the noisy coefficients. Now in [1] spatial domain filtering is used for the purpose of de-noising and in [2] transformed domain is used for image de-noising which shows improved results than spatial domain filtering. In transformed domain filtering, wavelets has superior results in image de-noising because it has some useful properties i.e. multi resolution analysis (MRA) and energy compaction. Instead of using spatial domain and Fourier domain the trend goes towards the wavelet transform domain. In wavelet domain the study shows that large coefficients of the images contains important features of the image and small coefficients mostly contains noise.
Thresholding is an easy way to drop small coefficients and the noise will be removed efficiently. Thresholding used in the proposed method.

In the past few years a large amount of literature emerged on signal de-noising and comparison using different wavelet transform. In 1990's wavelet has been widely used in many fields of applications containing statistics estimation solving mathematical differential equations, Density estimation, image de-noising and compression. In 1995, Dohono and Johnstone invented a method of wavelet shrinkage which shows good results for 1-D signal de-noising and inverse problem solving [3]. These methods failed to meet improve the removal of noise from images. In de-noising and compression many of the coefficient values are dropped which are below the threshold value. The selection of the threshold has a great impact on the output image. Various methods are used to set a suitable optimal threshold value for the image thresholding [4, 5] but still the suitable optimal threshold value is big problem. Dohono and Johnstone invented universal thresholding for the optimal threshold value selection. The method finds a threshold value globally which is high value which drops a lot of useful information. The best threshold value is still a problem and challenge for researchers.

The wavelet transform will give us the translated and shifted version of the input image. The wavelet transform has time-frequency localization property. The shifted versions are frequency sub-bands which is used for the reconstruction of wavelet. During reconstruction it can restore the fine details of the input image and delete the unwanted coefficients of the noisy image. Different wavelet families are used for decomposition and reconstruction. The most recent and useful wavelet family is bi-orthogonal wavelet version 6.8. The comparison of orthogonal to bi-orthogonal wavelet family shows that bi-orthogonal has superiority in digital image processing. The bi-orthogonal wavelet has equal orthogonally and symmetry [6]. 
The gray scale images are actually composed of red, green and blue (RGB) color image but is presented in gray scale. In this paper we have used gray scale images for de-noising. By using statistical thresholding methods for de-noising and compression using two dimensional (2-D) discrete wavelet transform (DWT). In order to remove the noise and to retain the important features wavelet thresholding method and scale de-noising method is used for image de-noising and compression. [7, 8, 9, 10] proposed thresholding methods for noise removal which are more effective and easy to use and widely implemented. However all the above techniques have many drawbacks such as they are non-adaptive, having artifacts and blur. In this paper statistical thresholding method is used which are more adaptive and based on the statistics of the image. We have used bi-orthogonal 6.8 wavelet family which shows improved results, high de-noising, compression and edge preserving.

The paper is organized in five sections. Section I is introduction. Section II is introduction to 2-D DWT. Section III is de-noising techniques and statistical thresholding methods. Section IV shows the results and simulated data. Section V is conclusion and future work.

\section{DISCRETE WAVELET TRANSFORM}

Let the image be represented by $\{f i j, i, j=$ $12 \ldots . N\}$ where $\mathrm{N}$ is power of 2 .consider it is corrupted by additive white Gaussian noise and one observes

$$
g i j=f i j+n i j i, j=12 \ldots N
$$

Where $\{$ nij $\}$ are independently and identically distributed (iid) as normal Gaussian distribution of $\mathrm{N}\left(0, \sigma^{2}\right)$ and is independent of $f i j$. The goal is to remove the noise or de-noise the noisy contaminated image $\{g i j\}$ and to obtain the approximated or estimated version of $\{f i j\}$.

Now let $\mathrm{G}=g i j$ and $\mathrm{F}=f i j$ and $\mathrm{N}=n i j$. The capital letters represents the matrix representation of the image which is under consideration. Now this is the representation of a noisy image. After passing this image from wavelet transform W. the equation becomes $\mathrm{Y}=\mathrm{W}_{\mathrm{g}}$ the wavelet transform of noisy image. Here $\mathrm{W}$ is the 2-D dyadic orthogonal wavelet transform operator. $\mathrm{W}_{\mathrm{f}}$ and $\mathrm{V}=\mathrm{W}_{\mathrm{n}}$ are the wavelet transform of the input image and the noise respectively. The readers are referred to $[11,12]$.for details of 2-D orthogonal wavelet transform. In the figure below 2-D DWT decomposition of input image occurs. It is very easy to label the sub-bands of the transform. The subbands are $\mathrm{HH}_{k}, \mathrm{HL}_{k}, \mathrm{LH}_{k}$, and $k=1,2 \ldots . j$ are called details. $\mathrm{HH}$ is diagonal details, $\mathrm{HL}$ are horizontal details and $\mathrm{LH}$ are vertical details. The low pass filters LL is called approximations, this contains the approximated coefficients values of the image. Here $k$ is the scale and $j$ is the level of decomposition. The sub-band at $\mathrm{k}$ scale has $\mathrm{N} / 2^{k} \times \mathrm{N} / 2^{k}$ size. Now the transform is orthogonal so the $\{\mathrm{Vij}\}$ is $\operatorname{iid} \mathrm{N}\left(0, \sigma^{2}\right)$.

The coefficients of the details sub band i.e. HHk, HLk, LHk are pass by wavelet thresholding method for finding the approximated or estimated output $\{\hat{f} i j\}$. The lowpass band i.e LL1 is called approximation at decomposition level 1. This section of the image is further decomposed into horizontal, vertical, and diagonal details. The coefficients of this approximation are kept. After passing the details from thresholding operator the estimated output is passed from inverse wavelet transform $\hat{f}=\mathrm{W}^{-1} \mathrm{x}$ where $\mathrm{W}^{-1}$ is the inverse wavelet transform operator.

\section{IMAGE DE-NOISING TECHNIQUES}

The most investigated domain in image de-noising using wavelet transform is nonlinear thresholding methods. Wavelet transform domain shows sparse property and wavelet maps noise from image domain to wavelet domain thus, the energy of the image is concentrated in high coefficients while noise energy is mostly in low coefficients values. This principle enables the separation of image important features from noise [13]. Now the procedure in which small coefficient values are dropped in large coefficients values left is known as hard thresholding but the drawback it produces visual artifacts. This is because of the unsuccessful attempt of removing large coefficients values. To overcome this problem soft thresholding was introduced. In this method the coefficient values shrinks towards the threshold value $T$. Most of the wavelet literature is about finding in optimal threshold value. Which can be adaptive or non-adaptive to the image. In wavelet thresholding there are two types which are mostly used for image de-noising and compression. One is soft thresholding and the other is hard thresholding:

Soft thresholding: It is also called shrinkage function. It shrinks the coefficient towards the threshold value $T$. it is a smoothing operator.

$$
\mathrm{D}(\mathrm{U}, \lambda)=\operatorname{sgn}(\mathrm{U}) * \max (0,|\mathrm{U}|-\lambda)
$$

Hard thresholding: It is the function which either keeps the coefficient or kills the coefficient value. The result of this technique have very sharp edge.

$$
D(U, \lambda)=\left\{\begin{array}{c}
U \text { for all }|U|>\lambda \\
=0 \text { otherwise }
\end{array}\right.
$$

\section{A. Universal Thresholding(Visu shrink)}

In the wavelet de-noising literature the universal thresholding is the most widely used one. It is globally approached and can be formulated as follows:

$$
\lambda_{T}=\sigma \sqrt{2 \log N}
$$

Where $\mathrm{N}$ is the size of the image and $\sigma$ is noise variance. The $\lambda_{T}$ must above the max level but not too large. Too much large coefficients may not be averted with increase in N Length and the threshold also increases due to Gaussian distribution.

Universal thresholding does not require prior information exactly like the Bayesian thresholding. For smooth data like Dohono it may be applied easily and conveniently.

When the size of the input signal is so large that it approaches to infinity the universal thresholding is the best candidate in that scenario. Also it is a good approach for statistical smoothness whose asymptotic behavior is better the mean square error.

This approach is too much fast and easy. Its implementation is straight forward, however when implemented on an image it produce a de-noised image which lost enough information 


\section{B. Statistical Thresholding Method 1}

In this method we find the mean of each detail sub-band ' $\mu$ '. The $\sigma_{y}$ is the variance of the degraded image which can be find by robust median estimator

$$
\sigma_{y}^{2}=[\operatorname{median}(\mid \text { each sub }- \text { band } \mid) / 0.6745]
$$

The noisy coefficients are very small and the signal coefficient are very large contains the useful information of the image. After the decomposition of the image to $\mathrm{N}$ level. The coefficients of the detail sub-bands are stored an array. Those values whose are greater than $2 \sigma_{y}, 3 \sigma_{y}$ are dropped and the other values are kept. i.e.

$$
\mathrm{y}>\left\{\begin{array}{c}
2 \sigma_{y}, 3 \sigma_{y} ; \mathrm{x}=2 \sigma_{y} \\
\text { Else } \mathrm{y}=\mathrm{y}
\end{array}\right.
$$

Finding the noise variance $\sigma_{n}$ and threshold value, finally add the value with mean ' $\mu$ '

$$
t=\sigma_{n}{ }^{2} / \sigma_{s}{ }^{2}
$$

\section{Satistical Thresholding Method 2}

The Statistical Thresholding method is effective for images including Gaussian noise. The observation model is expressed as follows:

$$
\mathrm{Y}=\mathrm{X}+\mathrm{N}
$$

Here $\mathrm{Y}$ is the wavelet transform of the degraded image, $\mathrm{X}$ is the wavelet transform of the original image, and $\mathrm{V}$ denotes the wavelet transform of the noise components following the Gaussian distribution $\mathrm{N}\left(0, \sigma_{n}{ }^{2}\right)$. Here, since $\mathrm{X}$ and $\mathrm{V}$ are mutually independent, the variances $\sigma_{y}{ }^{2}, \sigma_{x}{ }^{2}$ and $\sigma_{n}{ }^{2}$ of $\mathrm{y}, \mathrm{x}$ and $\mathrm{n}$ are given by

$$
\sigma_{y}^{2}=\sigma_{x}^{2}+\sigma_{n}^{2}
$$

It has been shown that the noise variance can be estimated from the first decomposition level diagonal sub-band $\mathrm{HH} 1$ by the robust and accurate median estimator [4] by (5).

The variance of the sub-band of degraded image can be estimated as:

$$
\sigma_{y}{ }^{2}=1 / M \sum_{m=1}^{M}(A m)^{2}
$$

Where Am are the wavelet coefficients of sub-band under consideration, $\mathrm{M}$ is the total number of wavelet coefficient in that sub-band. The statistical thresholding method 2 technique performs soft thresholding, with adaptive data driven, sub-band and level dependent near optimal threshold given by

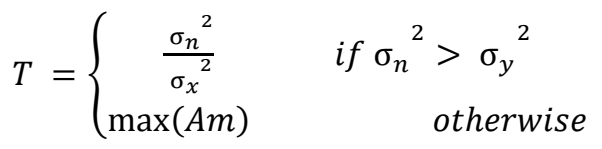

\section{Simulation RESUlts}

To evaluate the performance of above techniques we applied it on different images. Five images Lena, Barbara, house mcslibrary and cameraman are used as test images. All the five images are of size $(512 \times 512)$ are applied to the above techniques at different standard deviation levels $\sigma=15$, 20,25,30,35. We investigated different wavelet families. $\mathrm{Bi}$ orthogonal 6.8 (Bior6.8) [6] wavelet has superior results. We applied bi orthogonal 6.8 (Bior6.8) wavelet and decomposition level five in our simulations.to check the performance we compared the results with hard thresholding, soft thresholding, visu shrink, statistical method 1 and statistical method 2 using Peak signal to noise ratio (PSNR)[16]

$$
\text { PSNR }=10 \log 10_{10}\left(\frac{(\max (\mathrm{f}(\mathrm{m}, \mathrm{n})))^{2}}{\operatorname{MSE}}\right)
$$

In the above equation $\mathrm{f}(\mathrm{m}, \mathrm{n})$ shows the input image. We are dealing here with gray scale images. So we have

$$
(\max (\mathrm{f}(\mathrm{m}, \mathrm{n})))=255
$$

Where MSE is the mean square error between the degraded image and original image formulated as below.

$$
\text { MSE }=\sum_{M N} \frac{f(m, n)-\tilde{f}(m, n)}{M \times N}
$$

The other parameter used for psych-visual comparison is structural similarity index (SSIM) [16] which shows the structural similarity of the two images. SSIM index is calculated between two images $\mathrm{X}$ and $\mathrm{Y}$ is formulated as:

$$
\operatorname{SSIM}(X, Y)=\frac{\left(2 \mu_{x} \mu_{y}+C 1\right)\left(2 \sigma_{x y}+C 2\right)}{\left(\mu_{x}+\mu_{y}+C 1\right)\left(\sigma_{x}+\sigma_{y}+C 2\right)}
$$

Here

$\mu_{x}$ is the average of $x$

$\mu_{y}$ is the average of $y$

$\sigma_{x}$ is the variance of $x$

$\sigma_{y}$ is the variance of $y$

$\sigma_{x y}$ is the covariance of $x y$

$C 1$ and $C 2$ are constants

For subjective analysis mean opinion score (MOS) is used from decades. MOS is a test that shows the human view about the quality of images. It is ranked from unacceptable to excellent in the number from 1 (worst) to 5 (best). It is the averaged value of many users opinion $[14,15]$. The table is shown below:

TABLE I. MEAN OPINION SCORE (MOS)

\begin{tabular}{|l|l|}
\hline MOS & QUALITY \\
\hline 1 & Unacceptable \\
\hline 2 & Poor \\
\hline 3 & Fair \\
\hline 4 & Good \\
\hline 5 & Excellent \\
\hline
\end{tabular}

The graph in figure 4 . is the PSNR versus noise variance for the image Lena shows that the statistical method 1 and statistical methods 2 performs better than visu shrink, soft and hard thresholding. As we increase the noise variance the PSNR value decreases. The plot shows the PSNR value of the noisy image along with existing and proposed techniques. Soft thresholding has improved the PSNR value to a little extent. Hard thresholding improved the result of soft thresholding. Visu shrink has improved the value of PSNR. Statistical 
thresholding method 1 and 2 both shows superior results than all other techniques.

Now the MSE (Mean square error) Plot of the image lena shows graph of noisy image whose values are very high. The lower the MSE values the greater noise is removed. The plot shows that the soft, hard and visu shrink has lowered MSE to some extent. The statistical thresholding method 1 has lowered the layer of MSE to bottom level. Statistical method 2 has crossed statistical method 1 in removing error form the image. All the method is shown in the graph below

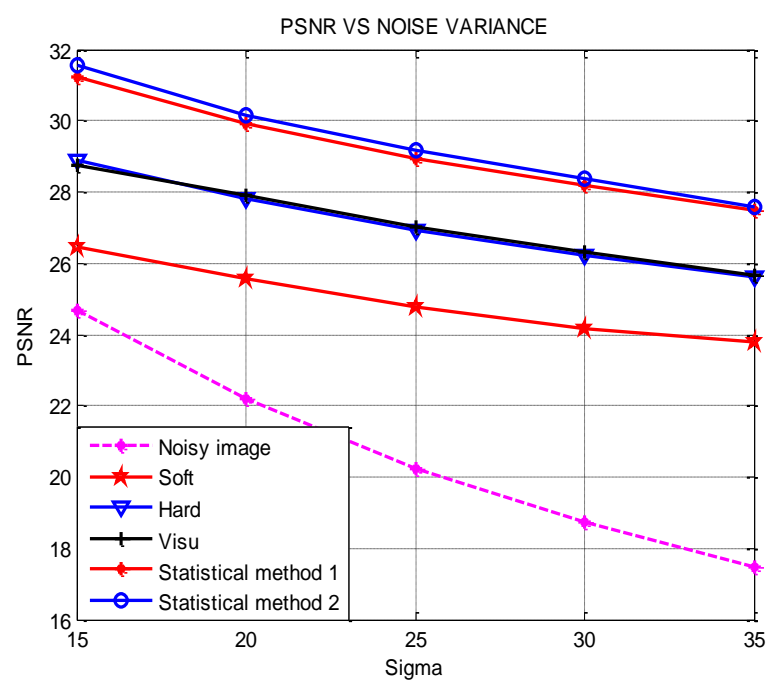

Fig. 1. Plot of PSNR vs Noise Variance for Sigma values=15, 20,25,30,35 for image Lena

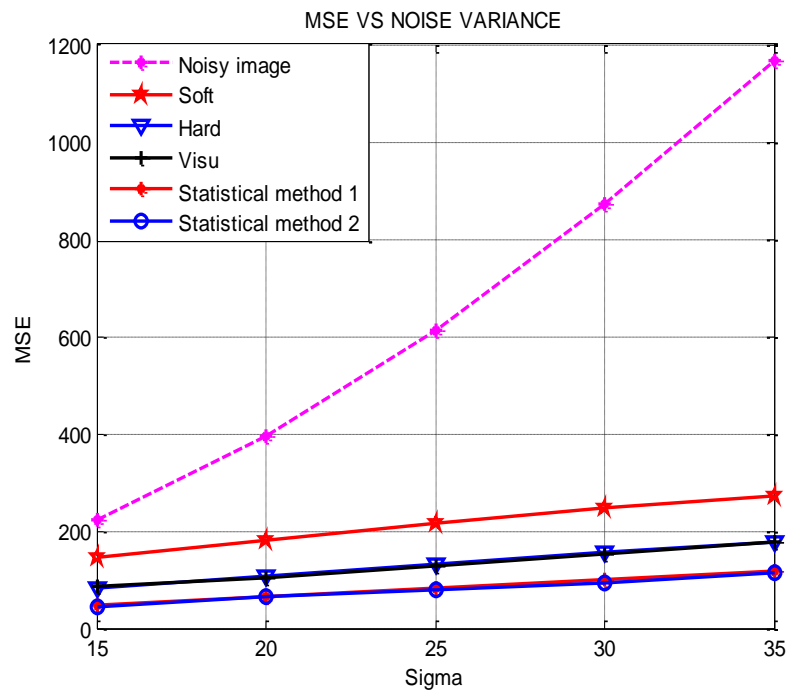

Fig. 2. Plot of MSE vs Noise Variance for Sigma values=15, 20,25,30,35 for image Lena

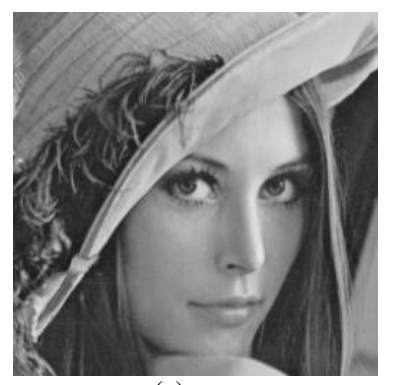

(a)

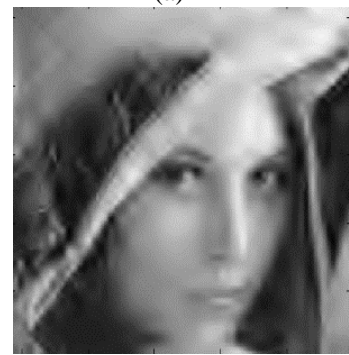

(c)

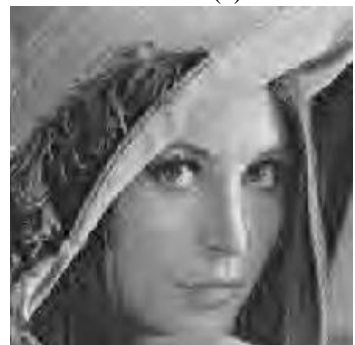

(e)

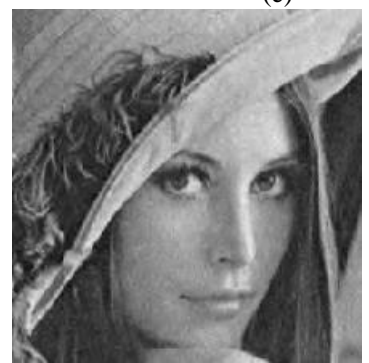

(g)

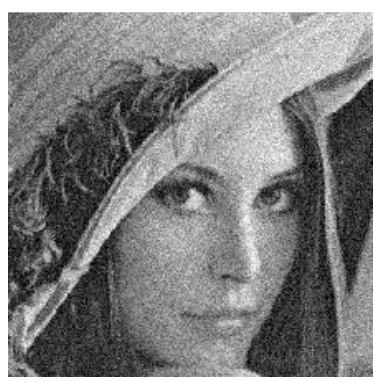

(b)

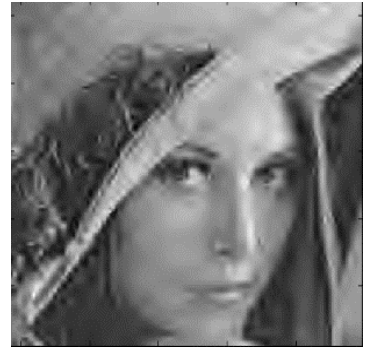

(d)

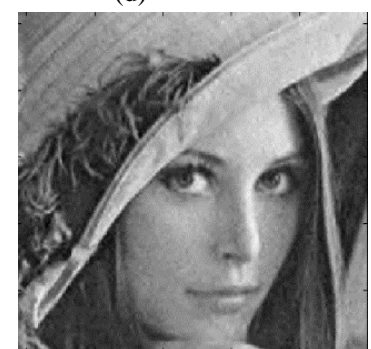

(f)
Fig. 3. (a) Original Lena image (b) Noisy Image with variance sigma $=20$ (c) Soft thresholding (d) Hard thresholding (e) visu shrink (f) Statistical thresholding method 1 (g) Statistical thresholding method 2

Visual quality of the images shows that soft thresholding has a blurred image but result is nearly a smoothed image as we know that soft thresholding gives us smoothed image. The result of hard thresholding is a sharp image having a large amount of artifacts. The visu shrink has improved result than hard thresholding. The statistical thresholding method 1 has more improved results than all previous method and removed artifacts and blur areas from the image. The statistical thresholding method 2 has improved the results of statistical method 2 and the visual quality is improved. The figure shows detail of all the methods. 
The SSIM (Structural similarity index) shows us a mean value and similarity map of the original and de-noised image. As the mean value approaches to 1 the noise approaches to zero. The SSIM map and mean values of soft thresholding method is very small shows up to $50 \%$ similarity to original image. The hard thresholding method shows $55 \%$ similarity visu $56 \%$, statistical method $161 \%$ and statistical method 2 shows $66 \%$ similarity of original ad de-noised image. The results of SSIM for the image of Military College of signals library MCS library is shown below.

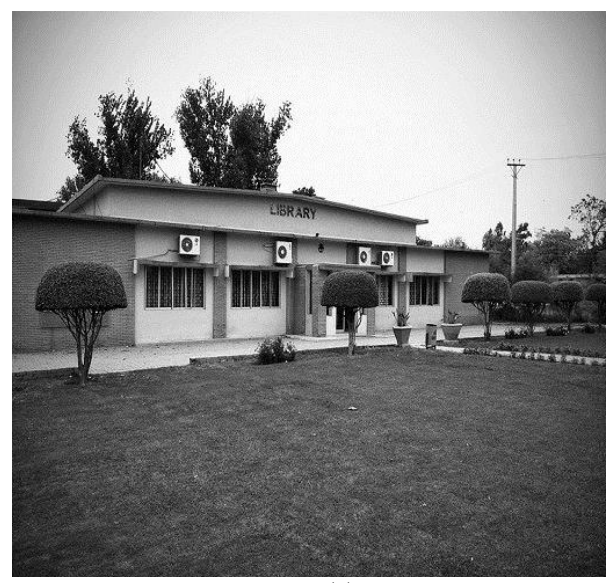

(a)

ssim Index Map - Mean ssim Value is 0.5072

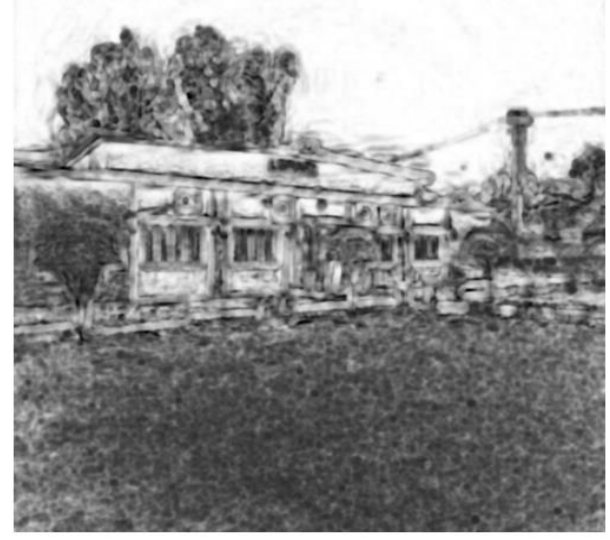

(b) ssim Index Map - Mean ssim Value is 0.5503

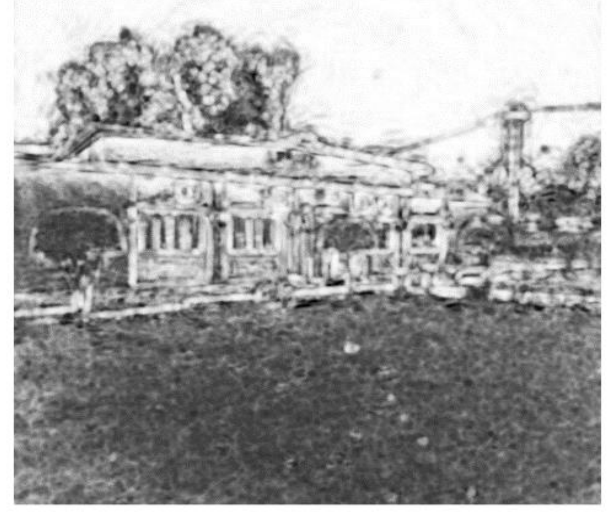

(c) ssim Index Map - Mean ssim Value is 0.5627

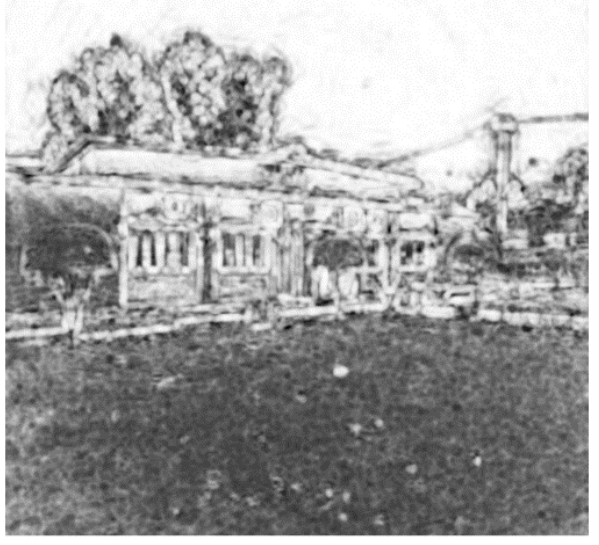

(d)

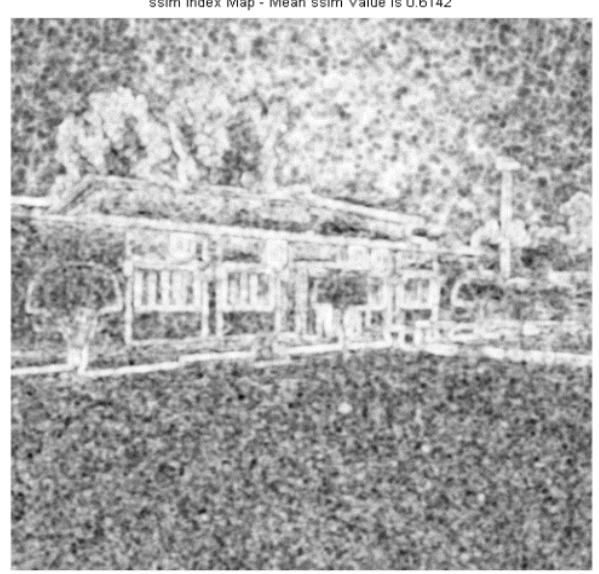

(e)

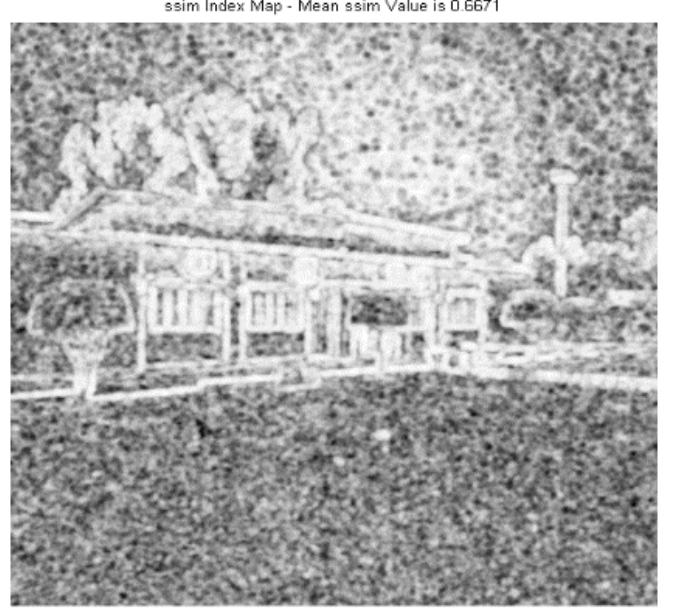

(f)

Fig. 4. (a) Original image of MCS library SSIM map for (b) soft thresholding (c) hard thresholding (d) Visu shrink (e) Statistical method 1 (f) Statistical method 2 
TABLE II. SSIM MEAN VALUE FOR DifFERENT METHODS FOR IMAGE MCS LIBRARY FOR NOISE VARIANCE 20

\begin{tabular}{|l|l|}
\hline Method & Mean value \\
\hline Soft thresholding & 0.5072 \\
\hline Hard thresholding & 0.5503 \\
\hline Visu Shrink & 0.5627 \\
\hline Statistical method 1 & 0.6142 \\
\hline Statistical method 2 & 0.6671 \\
\hline
\end{tabular}

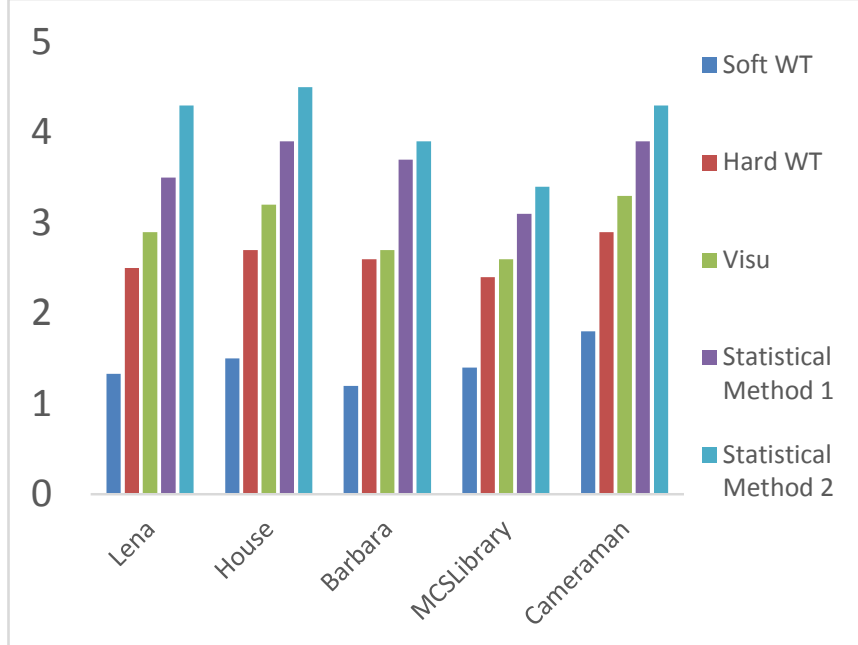

Fig. 5. Average value graph of mean opinion score for five images passed by five techniques

Fig. 5 shows an averaged value of MOS. The statistical thresholding method 2 has greatly minimized the noisy coefficients so the subjective quality for this technique is high while the other methods are arranged in the decreasing way showing his own image quality.

\section{CONCLUSION}

Image de-nosing and compression is the basic application of 2-D DWT. We have applied statistical thresholding methods on different images. We concluded that many thresholding methods used are not suited well for de-noising gray scale images. The results shows that for existing techniques many of the useful information of the image is lost which shows that degraded quality. The visual quality measured by MOS shows that existing image de-noising techniques produces blurriness and artifacts in the image. The statistical thresholding method 1 and statistical thresholding method 2 has largely improved PSNR values and MSE. The statistical thresholding methods also shows a higher structural similarity SSIM mean value. Statistical method 1 and statistical method 2 are adaptive and deals with each and every sub-band and coefficient value. The previous methods were level dependent thresholding methods but were non adaptive. Statistical thresholding methods are more effective due to adaptive and coefficient relevance judgment. In future work we will apply these thresholding methods on true color image.

\section{FUTURE WORK}

In the future, the work done here can be extended in many directions. This work can be extended to analyze those images corrupted by salt and speckle noise, pepper noise and other noise models. The benefit of DWT is give visually a pleasing image and improves PSNR values. The work can be extended in such a manner to reduce the noise with no loss of actual information. Research can be done on different image to choose a suitable mother wavelet for de-noising and compression images effectively. The methods applied in this paper can also be extended to RGB images as well as video sequences.

\section{ACKNOWLEDGMENT}

The authors would like to thank editors and reviewers for their useful comment. This research was supported by Image processing center National University of engineering and technology (NUST) Islamabad, Pakistan.

\section{REFERENCES}

[1] A. Sharma and J. Singh, "Image de-noising using spatial domain filters: A quantitative study," Image and Signal Processing (CISP), 2013 6th International Congress on, Hangzhou, , pp. 293-298. 2013 doi: 10.1109/CISP.2013.6744005

[2] T. Shah, G. Shikkenawis and S. K. Mitra, "Epitome based transform domain Image De-noising," Advances in Pattern Recognition (ICAPR), 2015 Eighth International Conference on, Kolkata, , pp. 1-6. 2015 doi: 10.1109/ICAPR.2015.7050652

[3] Iain M.Johnstone David L Donoho. Adapting to smoothness via wavelet shrinkage.Journal of the Statistical Association,90(432):1200-1224, Dec 2007

[4] K. I. Kim, and Y. Kwon, Example-based Learning for Single-Image Super-Resolution and JPEG Artifact Removal. Technical Report No. TR-173, Max Planck Institute for Biological Cybernetics, August 2008.

[5] M. Mastriani y A. Giraldez, "Microarrays denoising via smoothing of coefficients in wavelet domain," WSEAS Transactions on Biology and Biomedicine, 2005

[6] M. Mastriani y A. Giraldez, "Fuzzy thresholding in wavelet domain for speckle reduction in Synthetic Aperture Radar images," ICGST International on Journal of Artificial Intelligence and Machine Learning, Volume 5, 2005

[7] M. Mastriani, "Denoising based on wavelets and deblurring via selforganizing map for Synthetic Aperture Radar images," ICGST International on Journal of Artificial Intelligence and Machine Learning, Volume 5, 2005

[8] M. Mastriani y A. Giraldez, "Kalman' Shrinkage for Wavelet-Based Despeckling of SAR Images," International Journal of Intelligent Technology, Volume 1, Number 3, pp.190-196, 2006.

[9] S. Grace Chang, Bin Yu and M. Vattereli. Spatially Adaptive Wavelet Thresholding with Context Modeling for Imaged noising. IEEE Transaction - Image Processing, volume 9, pp. 1522-1530. 2000.

[10] Maarten Janse. Noise Reduction by Wavelet Thresholding. Volume 161, Springer Verlag, United States of America, I edition. 2001.

[11] J. Chen, C. Tang, and J. Wang. Noise brush: interactive high quality image-noise separation. ACM Trans. Graphics, 28(5), 2009.

[12] Z. Wang, A. C. Bovik, H. R. Shiekh and E. P. Simoncelli,'Image quality assessment: From error visiability to structral similarity," IEEE Transaction on Image processing, vol 13, no. 4, pp.600-612, Apr. 2004

[13] Atidel Lahouhou, Emmanuel Viennet and Azeddine Beghdadi Selecting Low-level Features for Image Quality Assessment by Statistical Methods Journal of Computing and Information Technology - CIT 18, 2010 , 